\title{
Heat of a single electron
}

\author{
Imaging resonant dissipation from individual atomic defects in graphene \\ Authors: Dorri Halbertal, Moshe Ben Shalom, Aviram Uri, Kousik Bagani, \\ Alexander Y. Meltzer, Ido Marcus, Yuri Myasoedov, John Birkbeck, Leonid S. \\ Levitov, Andre K. Geim, Eli Zeldov \\ Science 358, 1303-1306, 2017
}

\begin{abstract}
Recommended with a Commentary by Anton Akhmerov, Kavli Institute of Nanoscience, Delft University of Technology
\end{abstract}

The reason why nanoelectronics is hard is the lack of information combined with the lack of control. Put simply, the best probe we often have is hooking up electrodes to a device and measuring the current that flows through it. Because the current is a sum over all the possible paths an electron takes from one electrode to another, what is happening within a device is at best an educated guess. A more sensitive technique, scanning gate microscopy (see [1] for a recent example) utilizes a small mobile gate electrode and tells us how electrons respond to a local change in electrostatic potential, providing information about the local compressibility or how many electrons emitted by an electrode reach each point. A similar tool, scanning SQUID magnetometry uses magnetic field sensitivity of a small superconducting ring to probe a local magnetic field, and then reconstruct the electric current distribution by inverting Biot-Savart law.

In their work Halbertal et al. report a new way of utilizing a scanning SQUID. By pumping Helium gas between the sample and the tip of the SQUID probe, they create a thermal coupling between the probe and the device. The high temperature sensitivity of the critical current carried by a small superconductor serves then as a probe of a local temperature increase. By itself this probe is already impressive: in a previous work by the same group [2], where the authors measure a heating of a single nanotube, the minimal visible feature size is less than $100 \mathrm{~nm}$, while the excess heat due to electric transport distributes over a scale of about a micron. This probe is also non-invasive, being able to measure excess sub-millikelvin heat on top of the base temperature of $4.2 \mathrm{~K}$, and producing a negligible back-action on the device itself due to a much lower heat conductivity than the substrate. Thanks to this extremely high sensitivity the authors are able to answer not only the question "where do the electrons go?", but also "where do the electrons create phonons".

The main new physical phenomenon reported in the paper is the observation of resonant inelastic scattering between electrons and localized atomic defects. Because a typical feature size of the excess heat is of an order of a micron, at first it appears to be impossible to attribute the energy loss to a single atomic defect. Such a measurement becomes possible because the same SQUID tip used for measuring the temperature also works as a local gate 
electrode, similar to a scanning gate. The size of this gate and its distance from the sample of less than $100 \mathrm{~nm}$, therefore enabling high resolution probing of the device. When this gate tunes the energy of a resonant scatterer to coincide with the energy of incident electrons, it enhances the inelastic scattering from that defect and increases the local temperature. Finally, because the coupling of the gate electrode to the scatterer depends on the distance, for every fixed gate voltage applied to a SQUID the resonance condition is fulfilled whenever the probe is at a fixed distance from the scatterer. The temperature scan then registers a ring of increased temperature centered around the defect: confusingly the structure of this ring is not due to the high spatial resolution of the temperature sensor, but rather due to it also functioning as a local gate.

Turning to the discovered physics, the authors' finding appear to be very reasonable in hindsight. First of all, their electrostatic simulations confirm that the response of resonant state energy to the gate matches that of a single state tuned by a local potential. The authors observe only a handful of such resonant scatterers within the graphene bulk, likely due to its high quality. On the other hand, the defect density at the graphene boundary is high, with the typical spacing of about $10 \mathrm{~nm}$ between nearest impurity states. Because graphene boundary is etched, it is likely to have irregular structure as well as inhomogeneous chemical passivation - in other words dirt, giving rise to multiple additional impurity states.

Observing the extra states near the boundary by itself is not surprising, however there are still several puzzling questions. Firstly, the spacing between different states is two orders of magnitude higher than interatomic distance, while the edge disorder is more likely to be present on every single atom. Additionally, there appears to be no level repulsion between nearby defects, contrary to what one would expect from regular impurities. Finally, because the authors probe only a narrow energy interval of a few millielectron volts around the charge neutrality point, it is surprising why they find a lot of these levels: an atomic defect would likely create an extra impurity state at the energy comparable to the lattice hopping, so electron volts. I believe the likely answer to these questions is rooted in the physics of the edge states in graphene - a low energy dispersive mode confined near the edge due to the coupling between momentum and sublattice provided by the graphene low energy dispersion.

The measurement itself also opens doors to further lines of inquiry. The additional knowledge about the resonant dissipation within a mesoscopic sample may lead us to revisit the Landauer picture of electric conduction where all the energy is lost in the electrodes far away from the sample. To answer this question, we would need to measure the energy balance and figure out the fraction of energy dissipated at the sample boundaries, rather than in the electrodes far away from the sample. Because we know the total dissipated heat from the $V \times I$ product, an extremely precise calibration of the scanning SQUID thermometer is required to compare the heating it observes to the total energy budget. Further, the scanning SQUID is blind to electron-photon coupling and radiative energy losses. Figuring out which loss type dominates and designing a tool that could probe more dissipation types simultaneously seems like an exciting research direction now that we know that we can learn a lot from measuring energy loss. Controlling and probing electron dissipation could then become a new tool in the condensed matter toolbox. 


\section{References}

[1] R. Steinacher, et al., arXiv:1709.08559 (2017)

[2] Dorri Halbertal, et al., Nature 539, 407-410 (2016) 